\title{
Comparison of Pyrolysis Behaviour Results between the Cone Calorimeter and the Fire Propagation Apparatus Heat Sources
}

\author{
PIERRE GIRODS ${ }^{1}$, NICOLAS BAL ${ }^{2}$, HUBERT BITEAU ${ }^{2,3}$, GUILLERMO REIN ${ }^{2}$, and \\ JOSÉ L. TORERO ${ }^{2}$ \\ ${ }^{1}$ LERMAB EA4370 \\ Nancy-Université \\ ENSTIB, 27 rue Philippe SÉGUIN - BP 1041 - 88051 Épinal Cedex 9 - France \\ ${ }^{2}$ BRE Centre for Fire Safety Engineering \\ University of Edinburgh \\ King's Buildings, AGB, Edinburgh EH9 3JL, UK \\ ${ }^{3}$ Exponent, \\ 17000 Science Drive, Suite 200, Bowie, MD 20175, USA
}

\begin{abstract}
The cone calorimeter and the fire propagation apparatus (FPA) are often used to carry out flammability studies of materials. There are various differences in the set-up of these two devices that could lead to different fire behaviour for the same material. Among these, the impact of the different heat sources used is studied here. The cone calorimeter employs an electrical cone heater and the FPA uses tungsten lamps to radiate a given heat flux level to the sample. Experiments are conducted in the FPA set-up using a conical heater or tungsten lamps as the heat source with clear PMMA and wood samples. Mass loss and temperature measurements are taken during the tests, and the bubble layer depth is measured after the tests. Significant differences in pyrolysis behaviour of both samples between the cone calorimeter and the FPA are consistently observed at the same heat flux level. These different pyrolysis behaviours can be explained by the wavelength dependency of the radiative material properties (reflectance, absorptance and transmittance). This conclusion is in agreement with, and provides an experimental confirmation, to theoretical findings in previous studies.
\end{abstract}

KEYWORDS: ignition, thermal degradation, material properties.

\section{NOMENCLATURE LISTING}

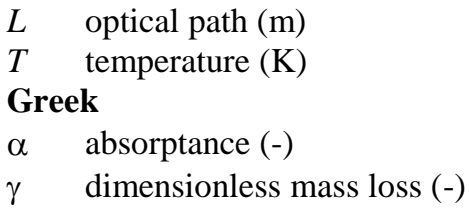

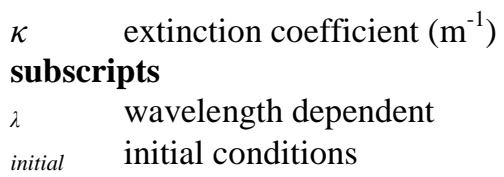

\section{INTRODUCTION}

Research in fire safety related to material characterization typically involves the use of bench-scale apparatus such as the cone calorimeter [1] or the FM Global fire propagation apparatus (FPA) [2]. Both apparatuses aim to provide similar information on the behaviour of materials exposed to an external heat flux [3,4]: ignitability (time to ignition, critical heat flux), heat release rate, combustion gases, mass loss and others. Accurate measurements of these variables are essential to understand the response of materials to fire and to obtain relevant flammability properties. Knowledge of the latter allows an advanced description of the chemical and physical mechanisms taking place during burning, i.e. modelling.

The design of the cone calorimeter [3] and the FPA [4] were defined more than 30 years ago. While the exhaust and gas collection systems are relatively similar, the heating system and the geometry of the combustion chamber contain significant differences. Under similar experimental conditions, there is an expectation that similar results will be obtained since both apparatuses were designed to be able to extract material properties. However, experimental measurements have shown otherwise.

The time to ignition measurements carried out with the cone calorimeter and the FPA (or Factory Mutual Research Corporation's Flammability apparatus and the Advanced Flammability Measurements Apparatus) 
for black polymethylmethacrylate (PMMA) samples, presented in Fig. 1, show two distinct groupings of data. The samples of black PMMA ignite faster with the cone calorimeter than with the FPA.

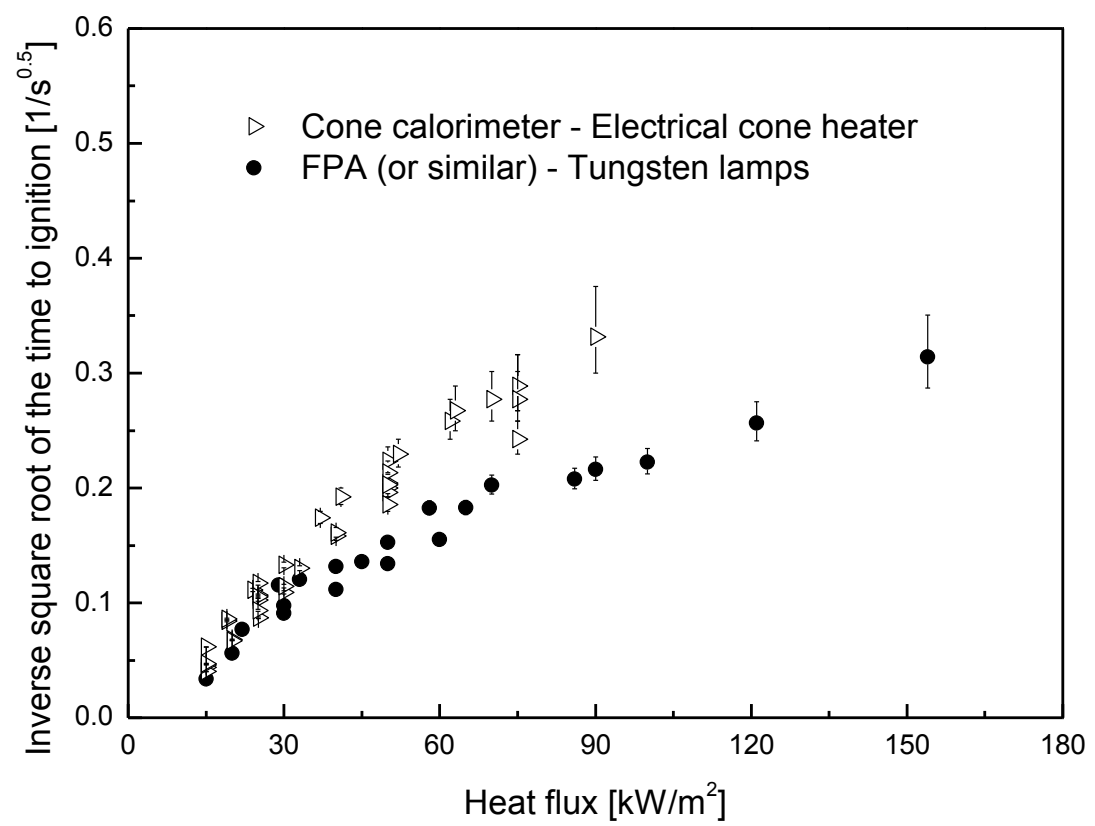

Fig. 1. Inverse square root of the time to ignition for black PMMA sample from literature review in [5].

The uncertainty is estimated at $\pm 2 \mathrm{~s}$.

Given the importance of the flammability properties when defining standard material classification or when using fire models, the origins of these differences need to be fully understood. The magnitude and consistency of the differences between the two test methods makes it unlikely that it stems only from error inherent to heterogeneities of the tested materials or from errors associated with the measurements. Consequently, potential biases relative to the methodology need to be investigated.

To be relevant, the argument has to emphasize the main differences between both apparatuses:

- The sample holder: it is square (stainless steel) for the cone calorimeter and square or circular (aluminium) for the FPA. Dimensions are nevertheless consistent.

- The design of the combustion chamber: different geometry.

- The pilot used for ignition: spark for the cone calorimeter and air/ethylene flame for the FPA.

- The heating source type: electrical heater wire with a truncated cone shape for the cone calorimeter and infrared tungsten lamps (24 tubular quartz lamps filled with halogen) for the FPA.

- The heating source position: directly above the sample for the cone calorimeter and on the side for the FPA.

- The experimental protocols: main difference linked to the presence of air supply (200 L/min recommended) blowing under the sample in the case of the FPA.

The equipment design and the experimental protocols of both apparatuses are explicitly mentioned in the corresponding standards: ASTM 1354-10a (cone calorimeter) [1] and ASTM E2058-03 (FPA) [2].

The degree of influence of these differences on the experimental results is best assessed on a one-to-one basis (uncoupled manner).

A non-exhaustive literature review [6-11] shows that the influence of the radiative characteristics of the heater source on the results is an area of concern.

Thomson and Drysdale [6] studied the impact of the spectral distribution on the time to ignition for PMMA using an electrical cone heater. In order to assess the impact, they used the same heater but with two 
different experimental protocols. First, they varied the heat flux by increasing and decreasing the temperature of the heater element while they kept the distance to the sample constant. Then, they carried out a new series of tests changing the heat flux level by varying the vertical elevation of the heater above the sample but with a constant heater temperature. According to Wien's law, variations in the emitter temperature have a direct influence on the spectral distribution and the position on the peak of emission. Thomson and Drysdale [6] concluded that ignition time measurements depend of the spectral distribution of the radiant heater. Finally, they compare their previous time to ignition to tests carried out with a $\mathrm{CO}_{2}$ laser beam providing a heat flux of $34 \mathrm{~kW} / \mathrm{m}^{2}$. The time measured with the laser was up to 6 times larger. In this work Thomson and Drysdale [6] focussed on the surface temperature at ignition and they found that for PMMA, the surface temperature tends to be independent of the emission wavelengths. They confirmed this with their laser tests. They compared their results with Kashiwagi [7] who also used a $\mathrm{CO}_{2}$ laser beam as the heater source. The time to ignition could not be compared since he performed his experiments at heat flux levels upper than $70 \mathrm{~kW} / \mathrm{m}^{2}$. However, the surface temperature measured was relatively higher. They claim that this discrepancy in the surface temperature measurements came from the pilot used and the fact that the part of radiation absorbed by the gas phase was significantly higher in the range of heat flux levels used by Kasiwagi [7].

Hallman [8] compared the ignition delay time for black, clear and white PMMA with two different heaters: a tungsten lamp and a benzene flame (see Fig. 2). The experiments were carried out with the sample mounted in the vertical position to reduce the dependence on the flow conditions. Hallman's results show that the impact of the heater does not appear with all the types of PMMA. Ignition of black PMMA (Fig. 2a) is independent of the heater used. However, the ignition of clear (Fig. 2b) and white PMMA samples is function of the heater source used and the difference in the delay time to ignition between both heaters increases with the flux level.

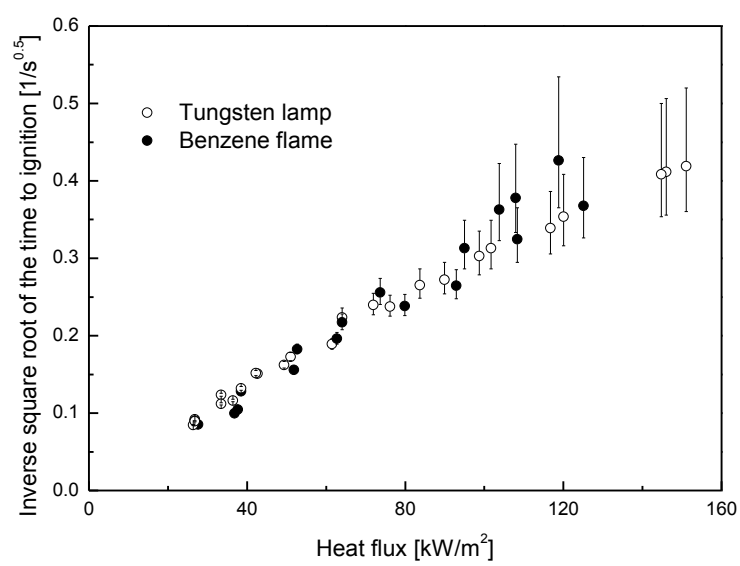

(a)

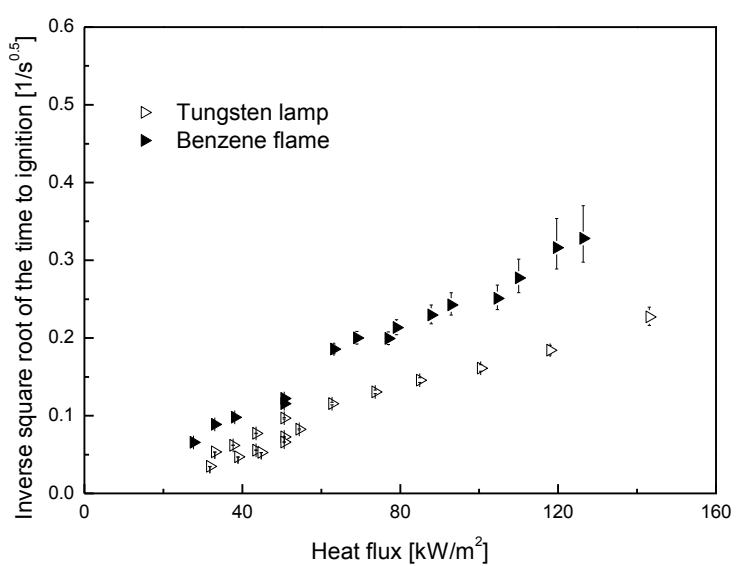

(b)

Fig. 2. Ignition test measurements for: (a) black PMMA; (b) clear PMMA with two heater sources: tungsten lamp and benzene flame [8]. The uncertainty is estimated at $\pm 2 \mathrm{~s}$.

Wesson et al. [9] carried out similar experiments to Hallman [8] (same apparatus, vertical orientation and same heaters) with the difference that they used wood samples (6 different types of wood for ignition tests). By taking into account the relative absorptance of their samples under benzene flame and tungsten lamps, and the effect of the density and the thickness of the different types of wood, they were able to explain the discrepancy between the ignition times measured with both heater sources for all wood types. They concluded that the time to ignition is strongly dependent on the spectral distribution of the incident radiation since the absorptance of wood is low (with a minimum of 0.16 ) between $0.6 \mu \mathrm{m}$ and $2 \mu \mathrm{m}$ and becomes higher (with a maximum of 0.95) at longer wavelengths. They presented absorptance measurements as a function of the wavelength for only two kinds of wood but they claimed that the results for the 14 types of wood tested are similar. They provided the averaged absorptance (across a range of wavelengths included between $0.3 \mu \mathrm{m}$ and $5.45 \mu \mathrm{m}$ ) for the 14 types of wood: the data spreads over a narrow range with an average value of $0.76 \pm 0.01$ for benzene flame radiation and $0.48 \pm 0.08$ for tungsten lamps. 
Recently, Försth and Roos [10] have carried out a large experimental study on absorptance measurements for different types of material. They assessed theoretically the impact of the heater temperature by calculating the effective absorptance of their sample as a function of the emissive power of the radiative source. They also provide some interesting results on the evolution of the absorptance of the sample after being exposed to an external heat flux for different times.

Linteris et al. [11] have studied the concept of the absorptance in detail and provide experimental results where they clearly show a dependency on thickness. On top of that, they show that according to the range of wavelengths analysed, the measurements of effective absorptance can give results differing by an order of magnitude. Their results emphasize the relative caution that experimentalist and modelling teams need when they deal with in-depth radiation absorption.

As a consequence of these studies, experimental teams [12-14] have resorted to using a black coating on all tested samples to guarantee that the heat flux measured by a gauge is the same as that absorbed by the material surface, independently of the radiative properties of the material. While this guarantees consistency between tests, it changes the impact of the effective radiative properties of the material and also results in non-quantified degradation issues of the coating. A recent study [5] tends to prove that a black coating on the exposed surface does not guarantee a total surface absorption.

The present work focuses on how the heating systems of the cone calorimeter and the FPA affect the pyrolysis behaviour of flammable materials. Experiments have been conducted using clear PMMA and wood (Spruce) samples. Comparisons of visual observations and mass loss and temperature measurements are performed. These complement previous experimental findings concerning the impact of the radiative source on the ignition delay times and confirm theoretical ones. Indeed, a deeper analysis of the pyrolysis behaviour (not only on delay time to ignition) enables to emphasize the agreement to theoretical findings on the impact of the heater source type.

\section{EXPERIMENTAL SECTION}

\section{Sample Preparation}

The present study incorporates several modifications to the standard sample holders to minimize the effect of these on the results. The main differences between both sample holders are associated with the insulation of the sample and the way the sample edges are treated. Therefore, for the present study, the sizes of the samples were reduced to $65 \times 65 \times 65 \mathrm{~mm}$ for the wood and $75 \times 75 \times 25 \mathrm{~mm}$ for the clear PMMA. The sample edges were treated in an identical manner. The lateral sides of the samples were insulated with ceramic fibres (10 mm width) coated with aluminium foil that also covered the edges of the sample. Heat transfer through the back of the sample was standardized by introducing an insulated aluminium block [15]. The back surface of the sample was thus in contact with an aluminium block that was insulated on the side and back (see Fig. 3). High thermal conductivity glue (Dow Corning 340) was added between the back surface of the sample and the aluminium block to ensure good contact. In the case of wood, the samples are placed such that the wood fibres are perpendicular to the vertical axis. More detailed justifications for the sample holder arrangement can be found in Carvel et al. [15]

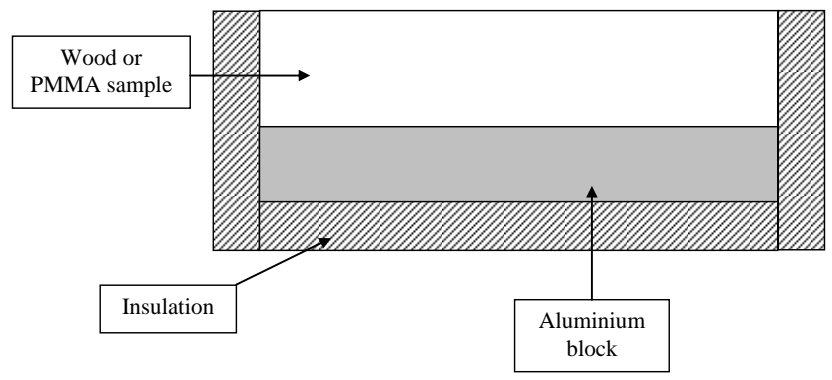

Fig. 3. Schematic of the sample holder. 


\section{Experimental Procedure}

Experiments have been performed with the two types of heaters (electrical cone heater and infrared tungsten lamps) at $20 \mathrm{~kW} / \mathrm{m}^{2}$. To make the tests comparable, they have been carried out both within the chamber of the FPA apparatus. In the case of the test with the conical heater, the FPA has been modified (see Fig. 4) by placing the electrical cone heater above the sample as it is in the cone calorimeter.

In the FPA configuration, 4 heaters composed of 6 tungsten tubular quartz lamps filled with halogen (Research Inc. model 5208) have been used. The conical heater is made of Inconel and its temperature is regulated by a PID (Proportional Integral and Derivative) temperature controller. Both heater types has been calibrated using a heat flux meter to insure that the radiant heat on the top surface of the sample is kept within $\pm 10 \%$ in time and space during the course of the experiment.

Natural convection conditions are chosen in order to reduce the perturbation of the flow caused by the presence of the electrical cone heater just above the sample. To increase the time before ignition, the tests have been performed without a pilot.
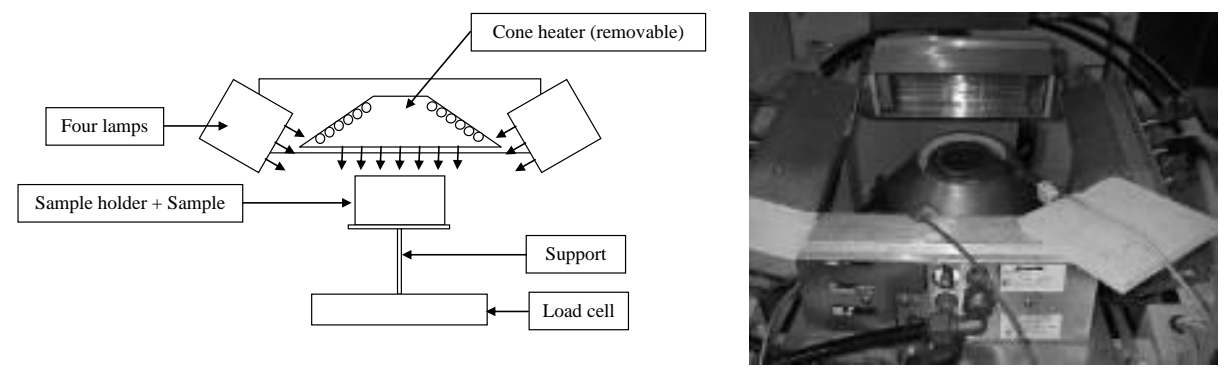

Fig. 4. Schematic and picture of the experimental apparatus with both heater types in their respective positions (removable).

The mass loss of the sample has been measured for both materials studied. The temperature profile has been recorded in-depth only for the wood samples. The measurements have been carried out with three Type-K thermocouples ( $1 \mathrm{~mm}$ of diameter) inserted parallel to the exposed surface at 5,15 and $25 \mathrm{~mm}$ depths. For the PMMA samples, only the temperature of the aluminium block has been measured. In-depth temperature measurements with clear PMMA were avoided because this material has a high transmittance and the measured temperature would correspond to a complex mixture of the absorption of the radiation by the thermocouple and conduction from the material, thus introducing further sources of uncertainty.

Temperature and mass loss were not taken simultaneously due to the large perturbation on the mass loss reading caused by the vibrations of the thermocouples wires. Basic visual observations were noted on the thickness of the bubbles layer appearing due to in-depth thermal degradation.

\section{Experimental Results}

For both heaters set-up at $20 \mathrm{~kW} / \mathrm{m}^{2}$, auto-ignition was not observed for both types of materials (wood and clear PMMA), thus the discussion concerns only the interaction between the heaters and the samples.

\section{Clear PMMA Samples}

The temperature increase of the aluminium block located at the back is shown in Fig. 5 for only 500 s over the more than $2000 \mathrm{~s}$ the tests lasted. The repeatability of the results for both heater types is excellent $\left( \pm 2{ }^{\circ} \mathrm{C}\right.$ over $35 \mathrm{~min}$ - error bars inside the symbols).

Mass loss measurements are shown in Fig. 6 based on the dimensionless mass loss parameter $\gamma$ which is defined as the mass lost since the beginning of the test divided by the initial mass (percentage). The samples exposed to the tungsten lamps do not pyrolyse as fast as the ones exposed to the conical heater.

During the experiments, bubbles appeared on the exposed side of the samples. After the tests, the samples were cut (Fig. 7) in order to observe the thickness of the bubble layer on the cross section. It is observed that the bubble layer is thicker when the sample is exposed to the tungsten lamps. This indicates that the in- 
depth temperature profile depends on the heater type. In the case of the conical heater, heating takes place on a thin layer close to the top surface. In contrast, clear PMMA seems to absorb energy at a greater thickness when it is exposed to the tungsten lamps.

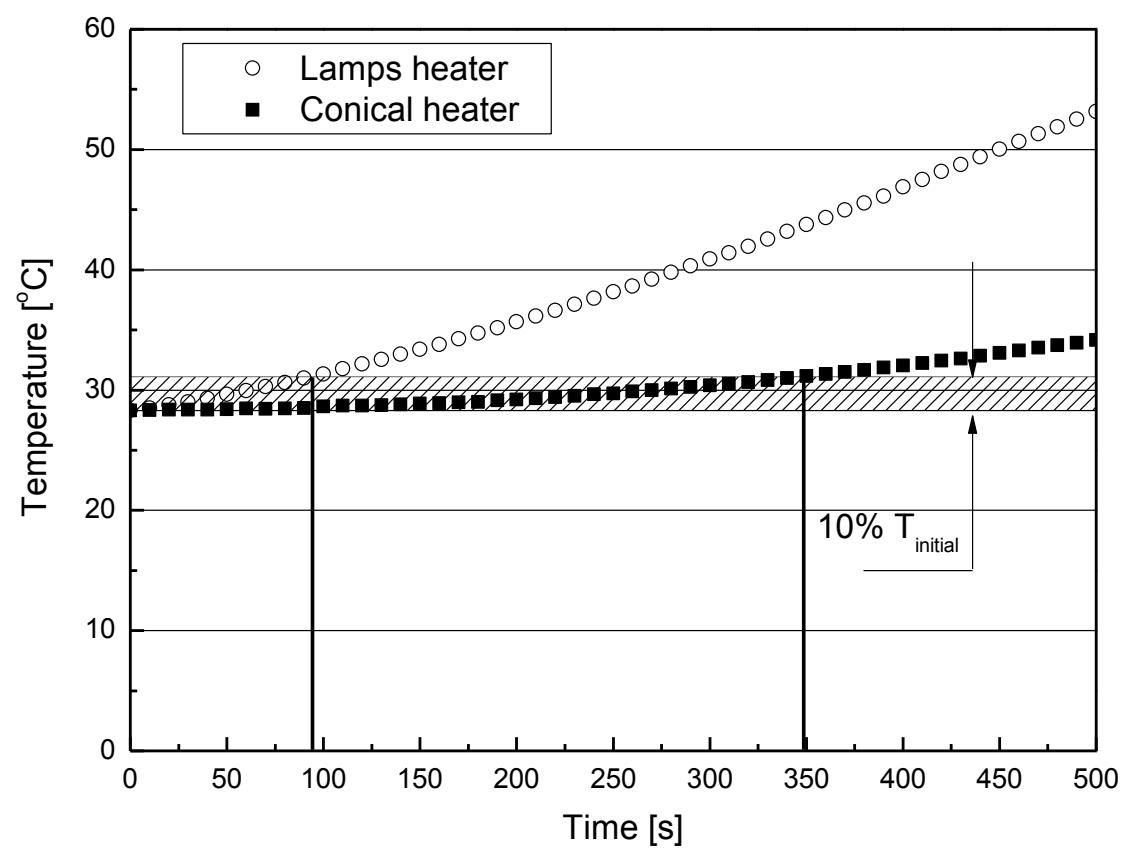

Fig. 5. Temperature increase of the aluminium block located at the back of the clear PMMA samples for experiments with tungsten lamps and conical heater.

The differences in sample observations, mass loss measurements and aluminium block heating are the result of different radiation attenuations according to the heater used. The non-reflected radiation in the clear PMMA surface is passing through the sample and it is subjected to in-depth attenuation as it is presented in Eq. 1. The energy is not absorbed at the surface but in-depth (Bouguer's law) and the value of the extinction coefficient $\kappa$ controlling this absorption is not the same for both heaters.

$\alpha_{\lambda}=1-e^{-\kappa L}$

A consequence of the in-depth radiation absorption is the presence of a peak of temperature inside the solid and not at the surface. This characteristic is more apparent as the heat flux level increases [5]. The peak location is related to the depth penetration by radiation which is proportional to the inverse of the extinction coefficient. The radiative penetration depth is independent of the heat flux level and is established instantaneously, whereas the thermal depth penetration by conduction grows at a rate decreasing with time. 


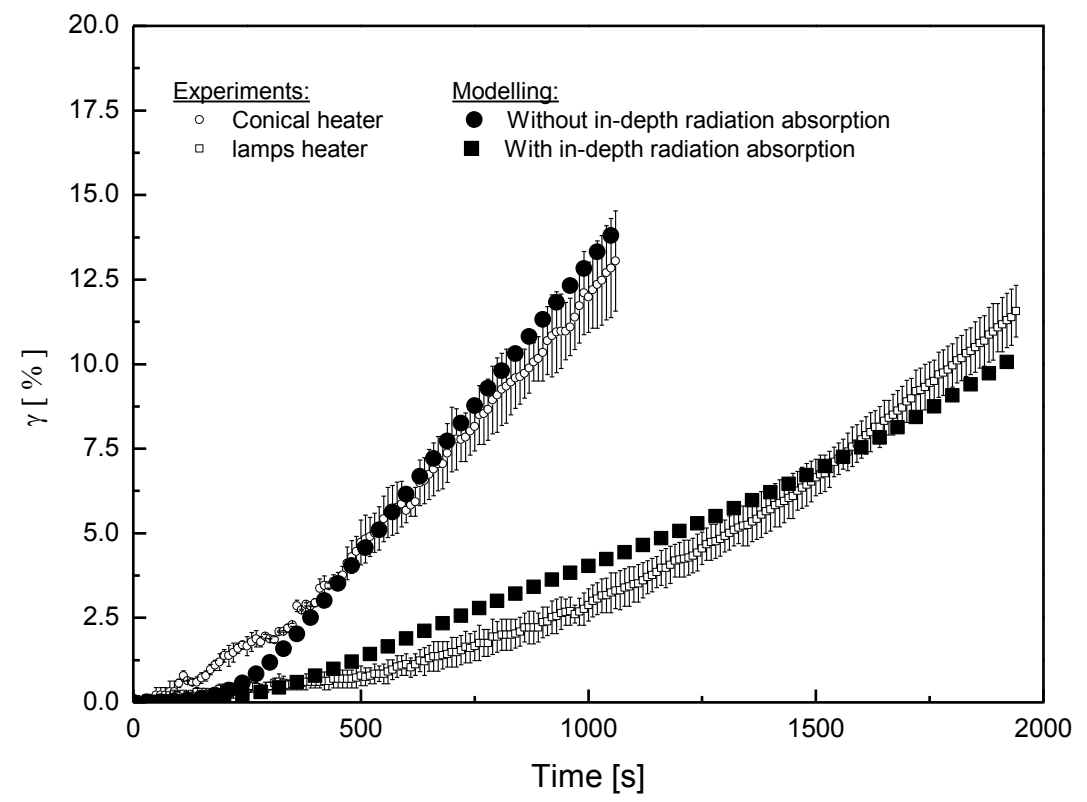

Fig. 6. Dimensionless mass loss coefficient $\gamma$ versus time for tungsten lamps and conical heater experiments with clear PMMA samples.

In order to estimate the value of the extinction coefficient $\kappa$, measurements of the transmittance of the clear PMMA (by means of a heat flux gauge) for two different sample thickness ( 5 and $25 \mathrm{~mm}$ ) have been carried out with the tungsten lamps. This experimental protocol correspond to the one operated by Jiang et al. in Ref. [16] for a range of wavelengths estimated between $0.46 \mu \mathrm{m}$ and $2.08 \mu \mathrm{m}(60 \%$ of the energy irradiated). The slope obtained by plotting the logarithm of the transmittance measured against the sample thickness gives an approximate value of $10 \mathrm{~m}^{-1}$ for $\kappa$.

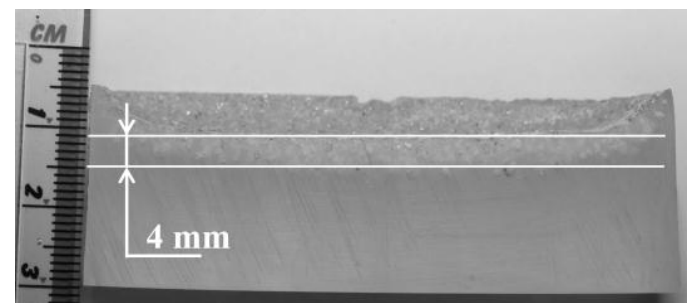

(a)

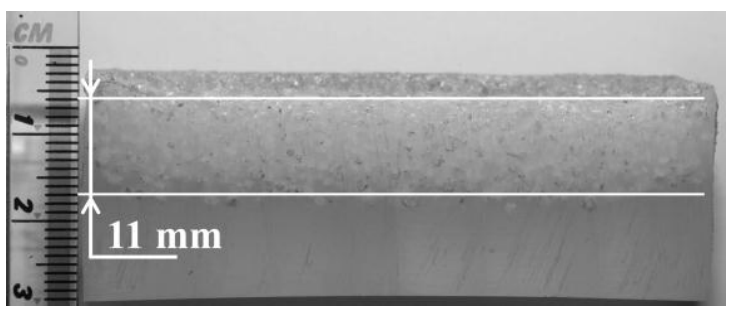

(b)

Fig. 7. Vertical sections of clear PMMA samples showing the bubble layer depth. Samples were exposed to a heat flux level of $20 \mathrm{~kW} / \mathrm{m}^{2}$ for 35 min with: (a) the conical heater; (b) the tungsten lamps.

In order to investigate deeper the heater dependence of the extinction coefficient, a qualitative analysis was performed with a pyrolysis model. This model, presented by Chaos et al. [17] solves kinetics, energy and mass conservation with the inclusion of the in-depth radiation absorption. The model is similar of the model GPYRO which is explained in details elsewhere $[18,19]$. For the qualitative analysis, a single step reaction following a first order Arrhenius law with only two species (solid $\rightarrow$ pyrolysis gas) is used.

Considering that the thin bubble layer thickness for the clear PMMA exposed to the conical heater indicates a high extinction coefficient $(\kappa \rightarrow \infty)$, it is first assumed that the material does not absorb in-depth but at the surface. Then, the most uncertain parameters value (kinetics parameters) have been modified in order to obtain numerically the same mass loss measured experimentally (see Fig. 6). The rest of the parameters are taken from Steinhaus [20]. In-depth radiation absorption is then included along with no other parameter values been changed. The measured value of $10 \mathrm{~m}^{-1}$ for the extinction coefficient with the tungsten lamps was used. By only adding in-depth radiation absorption, the mass loss measurements for samples exposed to the tungsten lamps is captured (see Fig. 6). While this modelling result is only approximate and the 
results should not be taken as quantitative, this simple analysis enables a confirmation that the in-depth radiation absorption mechanism is important and depends on the nature of the emission from the heaters.

\section{Wood Samples}

During the tests with wood samples, thermocouples were used to measure the temperature profile of the samples (see Fig. 8). The repeatability of the wood tests is worse when compared to the aluminium block measurements but this was predictable considering the non-homogeneity of the material properties (e.g. thermal conductivity) of the wood samples.

Among the temperature measurements plotted in Fig. 8, the temperatures $5 \mathrm{~mm}$ below the surface of the samples present the higher difference between both types of heaters. The samples exposed by the conical heater have the highest temperature at $5 \mathrm{~mm}$ under the surface.

The mass loss measurements for the wood samples (see Fig. 9) present the same behaviour than the ones presented in Fig. 6 for clear PMMA. Once again, the samples exposed to the conical heater have a mass loss at similar time which is more important than the ones exposed to the tungsten lamps. From Figs. 6 and 9 , it can be seen that the uncertainty on the measurements performed with the conical heater are higher. This seems to be due to the rate of the mass loss which is more important for the samples exposed to the cone calorimeter.

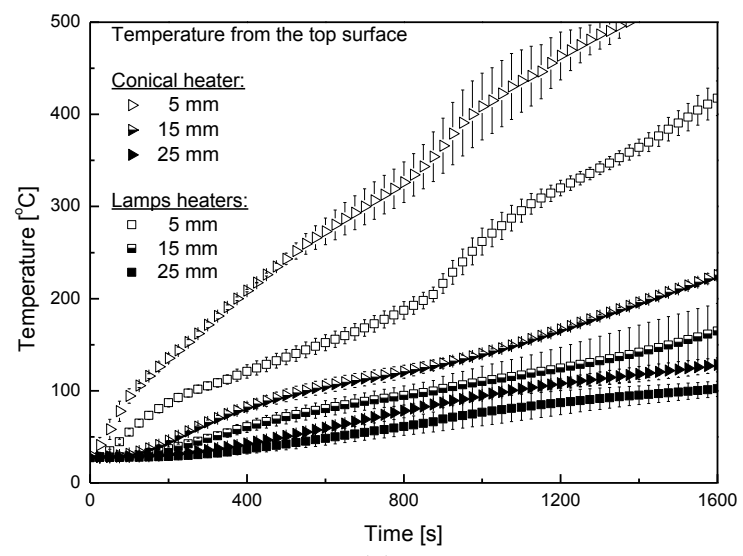

(a)

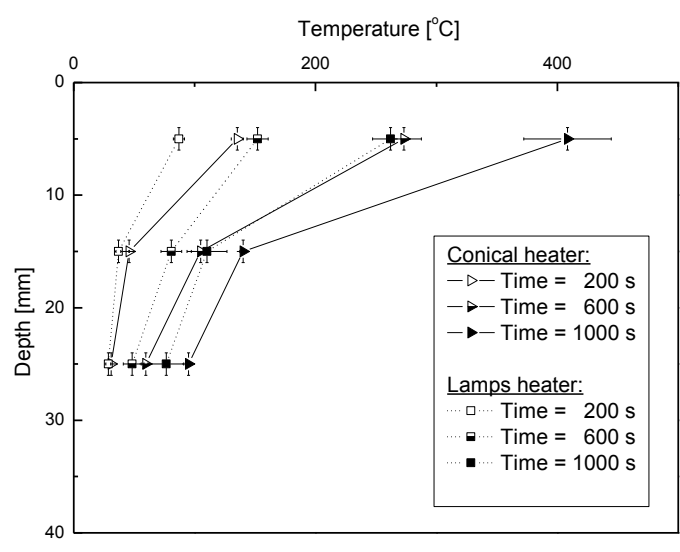

(b)

Fig. 8. Temperature measurement in-depth $(5,15$ and $25 \mathrm{~mm}$ below the exposed surface) for wood samples exposed to tungsten lamps and conical heater: (a) temperature vs. time; (b) depth vs. temperature.

\section{DISCUSSION}

In order to assess the possible causes of the discrepancy in the observed pyrolysis behaviour of the same material exposed by two different heat sources, the differences in the experimental set-up have to be investigated deeper.

The first one is link to the position of the source. In the case of the cone calorimeter the heater is located just above the sample (at $2.5 \mathrm{~cm}$ [1] - see Fig. 4). This could have an impact for two reasons: the presence of an obstacle perturbs the flow and the high temperature of the conical heater elements (see Table 1) creates buoyancy. In the case where tungsten lamps are used, there is no obstacle directly above the sample. In order to reduce the impact that the flow could have on the results, it has been decided, given the protocol presented in Refs. [1,2], to perform the test in a horizontal position but without any injected air (natural convection). The ideal solution to avoid this perturbation would be to use a vertical orientation $[8,9]$. 


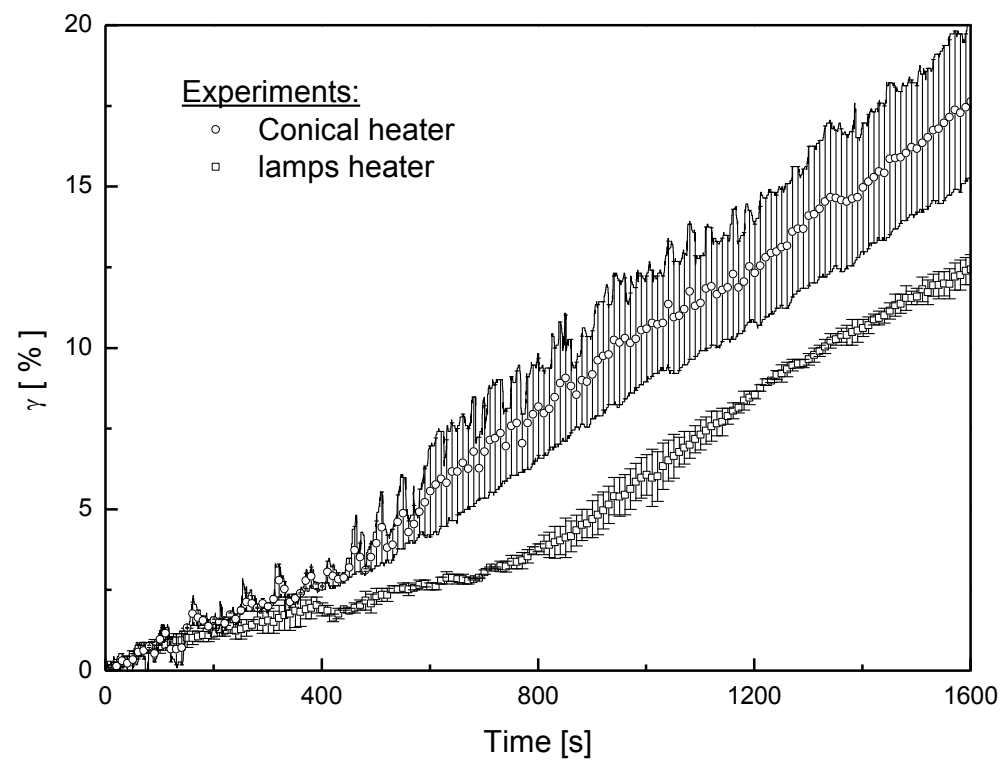

Fig. 9. Dimensionless mass loss coefficient $\gamma$ versus time for tungsten lamps and conical heater experiments with wood samples.

The second difference which is significant is the temperature range of the radiative source. Indeed, the conical heater temperature is lower than $1000 \mathrm{~K}$ for heat flux levels under $40 \mathrm{~kW} / \mathrm{m}^{2}$ whereas the tungsten lamps (for the FPA) are working in a temperature range greater than $2000 \mathrm{~K}$ for heat flux levels above $10 \mathrm{~kW} / \mathrm{m}^{2}$ (see Table 1$)$.

Table 1. Operating temperature for heat sources for cone calorimeter and FPA.

\begin{tabular}{|c|c|c|}
\cline { 2 - 3 } \multicolumn{1}{c|}{} & Electrical cone heater & Infrared tungsten lamps \\
\hline Heat flux $\left(\mathbf{k W} / \mathbf{m}^{\mathbf{2}}\right)$ & Temperature $(\mathbf{K})$ & Temperature $(\mathbf{K})$ \\
\hline 10 & 725 & 1970 \\
\hline 20 & 855 & 2280 \\
\hline 40 & 1013 & 2625 \\
\hline
\end{tabular}

It is reasonable to assume that these heat sources radiate approximately as blackbodies. Planck's distribution of blackbody emissive power [21] gives the temperature and wavelength dependency of the emissive power. From this, it is shown that the difference in the operating temperature range of the heaters changes significantly the wavelength range at which the emissive power is distributed (Fig. 10).

Hallman [8] and Wesson et al. [9] measured respectively the absorptance of PMMA and wood. Figure 10 compares the spectral distribution of the absorptance for clear PMMA (a-b) and wood (c-d) with the emissive power distribution as a function of the wavelength for the conical heater (a-c) and the tungsten lamps (b-d). For both materials, the absorptance is relatively high in the operating wavelength range of the conical heater and relatively low for the range of the tungsten lamps. In general, clear PMMA and wood absorbs energy mostly for wavelengths higher than $2 \mu \mathrm{m}$.

Försth and Roos [10], in their recent study, confirmed this global trend for wood and plastic samples (sometimes with a decrease between $4 \mu \mathrm{m}$ and $6 \mu \mathrm{m}$ ). Their numerical results of the effective absorptance over the emitting ranges of different heater temperatures corroborate the global observation explained above concerning the difference of absorption between radiation emitted at wavelengths below and above $2 \mu \mathrm{m}$. 


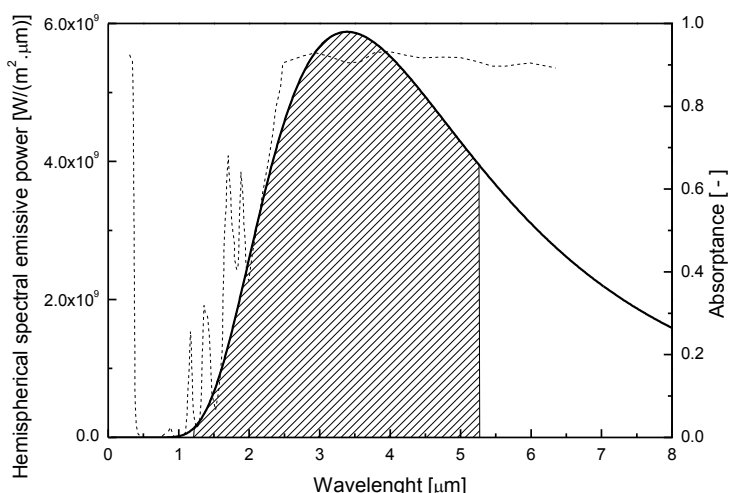

(a)

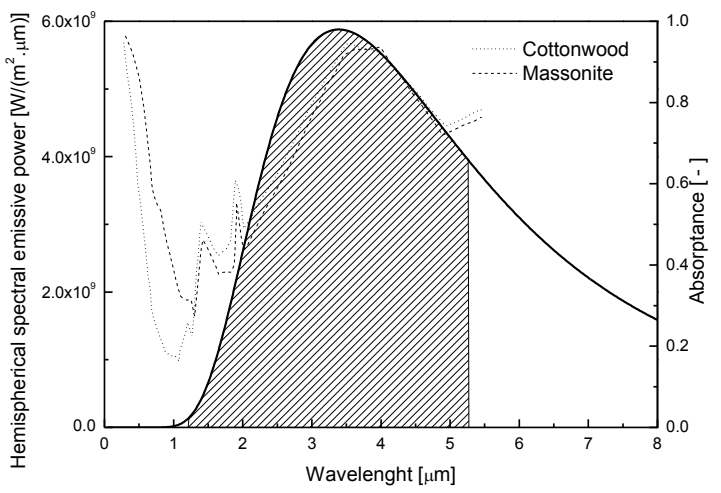

(c)

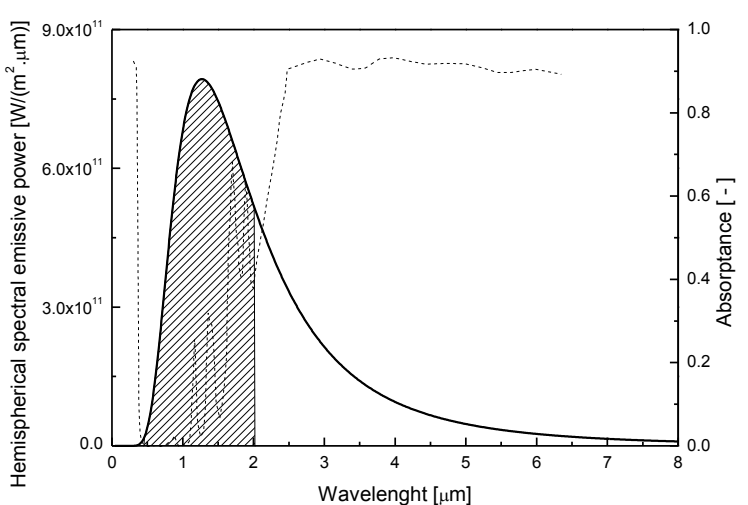

(b)

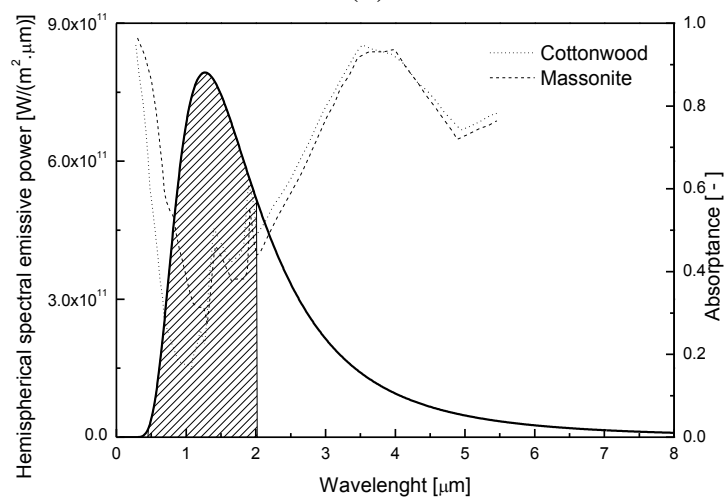

(d)

Fig. 10. Spectral distribution of the absorptance as measured in $[8,9]$ and of the emissive power of a blackbody at the corresponding heater temperature providing an heat flux of $20 \mathrm{~kW} / \mathrm{m}^{2}$ : (a) conical heater, clear PMMA; (b) tungsten lamps, clear PMMA; (c) conical heater, wood; (d) tungsten lamps, wood.

Shaded area is $60 \%$ of the total intensity and centred on the peak emissive power value.

In [8], Hallman indicates that he measured a non-negligible transmittance at low wavelengths for his clear PMMA samples (see Fig. 11).

From Figs. 10 and 11, it is seen that for clear PMMA, not all the incident radiation is absorbed by the sample and the transmitted part is high for wavelengths lower than $2 \mu \mathrm{m}$. For wood, the transmittance is very low (for the whole spectrum) and can be assumed equal to 0 . As a consequence, the low absorptance at wavelengths below $2 \mu \mathrm{m}$ means that a large fraction of the incident radiation is reflected [9]. These two different radiation behaviours explained well the measurements presented here.

For clear PMMA, the relatively high increase in temperature of the aluminium block when the tungsten lamps are used (wavelength below $2 \mu \mathrm{m}$ ) is due to higher transmission of the incident radiation. This high ratio of transmitted radiation means that the extinction coefficient $\kappa$ is low which is confirmed by the order of magnitude found by measurement: $10 \mathrm{~m}^{-1}$. In addition, a low coefficient $\kappa$ leads to a larger radiation penetration depth (confirmed by the visual thickness of the bubble layer in Fig. 7). The presence of bubbles indicates the thermal decomposition of the solid into pyrolysis gases. However, the mass loss rate is lower for the samples exposed to tungsten lamps in Fig. 6. This is the consequence of a lower amount of energy absorbed but also of wider distribution inside the sample.

The presence of the bubbles in-depth is opposite to the main hypothesis of most of the pyrolysis models which state that pyrolysis gases are instantaneously released out of the sample. This observation tends to show that the transport of the pyrolysates may play an important role. 


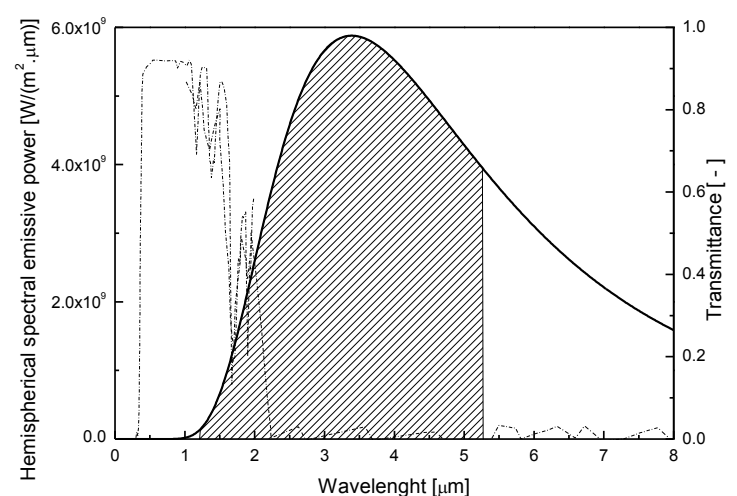

(a)

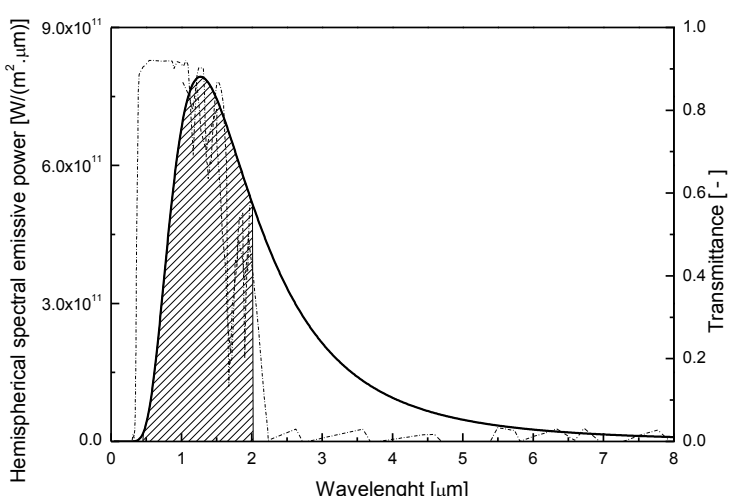

(b)

Fig. 11. Spectral distribution of the transmittance of clear PMMA as measured in [8] and of the emissive power of a blackbody at the corresponding heater temperature providing a heat flux of $20 \mathrm{~kW} / \mathrm{m}^{2}$ : (a) conical heater; (b) tungsten lamps. Shaded area is $60 \%$ of the total intensity and centred on the peak emissive power value.

For the wood, higher temperature measurements, just below the surface, are observed when the conical heater is used (wavelength above $2 \mu \mathrm{m}$ ). This is explained by the significant part of the incident radiation being reflected by the wood sample exposed to the tungsten lamps. The higher mass loss attributed to the samples exposed to the conical heater in Fig. 9 is therefore only the consequence of a higher quantity of energy absorbed and not of the thickness through which the energy is distributed.

Finally, the third difference between the two heaters sources studied here is linked also to the position of the heaters. Due to their respective position (Fig. 4) the incident radiation does not arrive with the same angle on the sample. The conical heater provides incident radiations intercepting the sample with a large range of angles (due to its inclined shape) whereas the infrared heater lamp radiations arrive with an angle between $70^{\circ}$ and $80^{\circ}$ to the normal of the surface. Hallman [8] studied the angular variation of the absorptance for clear PMMA and showed that the variation from a normal orientation to $70^{\circ}$ is included within $15 \%$ for wavelength lower than $2 \mu \mathrm{m}$. A supplementary study, with the relation given by Jiang et al. [16] to calculate the reflectivity at a particular wavelength (linked to reflective index), shows that the reflected ratio varies between 0.038 and 1 when the angle formed with the normal of the sample is between 0 and $90^{\circ}\left(0.17\right.$ for an angle of $70^{\circ}$ and 0.39 for an angle of $\left.80^{\circ}\right)$.

According to the experimental results from Hallman and from Försth and Roos [8,10], the absorptance of the black PMMA is approximately constant over the wavelength range covered by the conical heater and the tungsten lamps. Therefore significant difference between these heaters should not appear. Figure $2 \mathrm{a}$ is in perfect agreement with this statement whereas Fig. 1 is not. The difference between these two sets of data is the experimental protocols. In Fig. 2, the experiments were carried out with only one apparatus in a vertical orientation to avoid any flow disturbance whereas in Fig.1, the measurements are obtained from cone calorimeter and FPA (or similar apparatuses) ignition tests.

The significant differences between the ignition delay times in Fig. 1 are not linked to the wavelength dependency of the material but could come from the flow field and the sample holder differences. In [12], Beaulieu claims according to measurements performed with black PMMA, that the shape and the thickness of the sample do not bring significant difference on the time to ignition but the cone calorimeter sample holder (due to conduction through it at its edges) and the flow are responsible of some discrepancies. The pilot used (flame or spark) seems not to cause a significant difference to the piloted ignition time since these two types of pilot are used indifferently with the cone calorimeter and the FPA (or similar) among the data set presented in Fig. 1.

\section{CONCLUSION}

The impact on the results obtained with a cone calorimeter and a fire propagation apparatus (FPA) heater source has been studied here. This paper is builds on previous work which studied the impact of the heater 
source on time to piloted ignition. It confirms and expands previous studies by including temperature, mass loss and pyrolysis observations for clear PMMA and spruce wood samples.

Planck's wavelength distribution shows that the electrical cone heater from the cone calorimeter emits most of its intensity in a range of wavelengths greater than $2 \mu \mathrm{m}$ whereas, whereas for the tungsten lamps from the FPA, the range is mainly situated under $2 \mu \mathrm{m}$. Moreover, it appears from previous studies on absorptance measurement (Hallman [8], Wesson et al. [9] and Försth and Ross [10]), that most of the energy absorbed by clear PMMA and wood samples is for wavelengths above $2 \mu \mathrm{m}$. However, different behaviour appears for clear PMMA and wood when the wavelength is lower than $2 \mu \mathrm{m}$ : clear PMMA transmits the energy whereas wood reflects it. Mass loss and temperature measurements confirm that this mechanism explains the significantly different pyrolysis behaviour observed in the experiments. Therefore, it appears important to pay careful attention to the radiative properties of the material under study before making conclusion from the experimental results.

It should be noted that in Ref. [2], it is recommended to use a quartz tube between the tungsten lamps and the sample. However, the tube filters the wavelengths greater than $2 \mu \mathrm{m}$. So with its use, the difference between the two heaters would be increased. Moreover, for the typical uses of the cone calorimeter and the FPA, when the experimentalist desires to increase the heat flux imposed on the sample, the temperature of the heater is increased. This increase of temperature could therefore have an impact according to the radiative property of the material.

Finally, it appears that although some material like black PMMA do not have a significant wavelength dependency in their radiation material properties, some small discrepancies appear between the cone calorimeter and the FPA ignition tests. These differences could be caused by many other variables and need to be explored in more detail.

\section{ACKNOWLEDGEMENTS}

The FPA used here was donated by FM Global together with support for the experimental work. The financial support from BRE Trust to N. Bal is well appreciated.

\section{REFERENCES}

[1] ASTM Standard "E1354-10a: Standard Test Method for Heat and Visible Smoke Release Rates for Materials and Products Using an Oxygen Consumption Calorimeter", ASTM International, West Conshohocken, PA, 2010, http://dx.doi.org/10.1520/E1354-10A.

[2] ASTM Standard "E2058-03: Standard Test Method for Measurement of Synthetic Polymer Material Flammability Using a Fire Propagation Apparatus", ASTM International, West Conshohocken, PA, 2003, http://dx.doi.org/10.1520/E2058-03.

[3] Babrauskas, V., (1984) Development of the Cone Calorimeter - A Bench-Scale Heat Release Rate Apparatus Based on Oxygen Consumption, Fire and Materials 8: 81-95, http://dx.doi.org/10.1002/fam.810080206.

[4] Tewarson, A., and Pion, R. F., (1976) Flammability of Plastics, I-Burning Intensity, Combustion and Flame 26: 85-103, http://dx.doi.org/10.1016/0010-2180(76)90059-6.

[5] Bal, N., and Rein, G., (2010) Numerical investigation of the ignition delay time of a translucent solid at high radiant heat fluxes, Combustion and Flame, Article in press, http://dx.doi.org/10.1016/j.combustflame.2010.10.014.

[6] Thomson, H.E. and Drysdale, D., (1987) Flammability of plastics I: Ignition temperatures, Fire and Materials 11:163-172, http://dx.doi.org/10.1002/fam.810110402.

[7] Kashiwagi, T., (1979) Experimental observation of radiative ignition mechanisms, Combustion and Flame 34:231-244, http://dx.doi.org/10.1016/0010-2180(79)90098-1.

[8] Hallman, J.R., Ignition characteristics of plastics and rubber, $\mathrm{PhD}$ thesis, University of Oklahoma, Norman, 1971. 
[9] Wesson, H.R., Welker, J.R., and Sliepcevich, C.M., (1971) The piloted ignition of wood by thermal radiation, Combustion and Flame 16:303-310, http://dx.doi.org/10.1016/S00102180(71)80101-3.

[10] Försth, M., and Roos, A., (2010) Absorptivity and its dependence on heat temperature and degree of thermal breakdown, Fire and Materials, Article in press, http://dx.doi.org/10.1002/fam.1053.

[11] Linteris, G., Zammarano M., Wilthan B. and Hansen L., "Absorption of thermal radiation by burning polymer" Proceedings of Fire and Materials 2011, Interscience Communications Ltd, San Francisco, California, Interscience Communications, 2011, pp. 559-570

[12] Beaulieu, P.A., Flammability characteristics at heat flux levels up to $200 \mathrm{~kW} / \mathrm{m}^{2}$ and the effect of oxygen on flame heat flux, PhD thesis, Worcester Polytechnic Institute, 2005.

[13] Tewarson, A., and Ogden, S. (1992) Fire behavior of polymethylmethacrylate, Combustion and flame 89: 237-259, http://dx.doi.org/10.1016/0010-2180(92)90013-F.

[14] Delichatsios, M.A., Panagiotou, T, and Kiley, F, (1991) The use of time to ignition data for characterizing the thermal inertia and the minimum (critical) heat flux for ignition or pyrolysis, Combustion and Flame 84:323-332, http://dx.doi.org/10.1016/0010-2180(91)90009-Z.

[15] Carvel R., Steinhaus, T., Rein, G., and Torero, J.L., (2011) Determination of the flammability properties of polymeric materials: A novel method, Polymer Degradation and Stability 96(3): 314319, http://dx.doi.org/10.1016/j.polymdegradstab.2010.08.010.

[16] Jiang, F., De Ris, J.L., and Khan, M.M., (2009) Absorption of thermal energy in PMMA by indepth radiation, Fire Safety Journal 44: 106-112, http://dx.doi.org/10.1016/j.firesaf.2008.04.004.

[17] Chaos, M., Khan, M.M., Krishnamoorthy, N., De Ris, J.L., and Sergey, B.D., "Bench-scale flammability experiments: Determination of materials properties using pyrolysis models for use in CFD fire simulations" Proceedings of Interflam 2010, Interscience Communications Ltd, Nottingham, England, 2010, pp.697-708.

[18] Lautenberger, C.W., A generalized pyrolysis model for combustible solids, PhD thesis, University of California, Berkley, 2007. http://escholarship.org/uc/item/7wz5m7dg.

[19] Lautenberger, C.W., Fernandez-Pello, A.C., (2009) Generalized pyrolysis model for combustible solids, Fire Safety Journal 44(6): 818-839, http://dx.doi.org/10.1016/j.firesaf.2009.03.011.

[20] Steinhaus S., Evaluation of the thermophysical properties of poly(methylmethacrylate): a reference material for the development of flammability test for micro-gravity environments, Master thesis, University of Maryland, 1999, http://www.era.lib.ed.ac.uk/handle/1842/2831.

[21] Siegel, R. and Howell, J., Thermal radiation heat transfer $4^{\text {th }}$ ed., Taylor and Francis 2002, chap 11, pp 419-500, ISBN 1-56032-839-8. 